TRANSACTIONS OF THE

AMERICAN MATHEMATICAL SOCIETY

Volume 349, Number 1, January 1997, Pages 413-428

S 0002-9947(97)01713-3

\title{
LINEAR ISOMETRIES BETWEEN SUBSPACES OF CONTINUOUS FUNCTIONS
}

\author{
JESÚS ARAUJO AND JUAN J. FONT
}

\begin{abstract}
We say that a linear subspace $A$ of $C_{0}(X)$ is strongly separating if given any pair of distinct points $x_{1}, x_{2}$ of the locally compact space $X$, then there exists $f \in A$ such that $\left|f\left(x_{1}\right)\right| \neq\left|f\left(x_{2}\right)\right|$. In this paper we prove that a linear isometry $T$ of $A$ onto such a subspace $B$ of $C_{0}(Y)$ induces a homeomorphism $h$ between two certain singular subspaces of the Shilov boundaries of $B$ and $A$, sending the Choquet boundary of $B$ onto the Choquet boundary of $A$. We also provide an example which shows that the above result is no longer true if we do not assume $A$ to be strongly separating. Furthermore we obtain the following multiplicative representation of $T:(T f)(y)=a(y) f(h(y))$ for all $y \in \partial B$ and all $f \in A$, where $a$ is a unimodular scalar-valued continuous function on $\partial B$. These results contain and extend some others by Amir and Arbel, Holsztyński, Myers and Novinger. Some applications to isometries involving commutative Banach algebras without unit are announced.
\end{abstract}

\section{INTRODUCTION}

Let $\mathbb{K}$ denote the field of real or complex numbers. For a locally compact Hausdorff space $X$, we denote by $C_{0}(X)$ the Banach space of all continuous $\mathbb{K}$-valued functions defined on $X$ which vanish at infinity, equipped with its usual supremum norm. If $X$ is compact, we write $C(X)$ instead of $C_{0}(X) . X \cup\{\infty\}$ denotes the Alexandroff compactification of $X$.

Let $A$ be a linear subspace of $C_{0}(X)$. We will denote by $\sigma A$ the set of all $x_{0} \in X$ such that for each neighborhood $U$ of $x_{0}$, there is a function $f$ in $A$ such that $|f(x)|<\|f\|$ for all $x \in X-U$. Let us define the set

$$
\sigma_{0} A:=\sigma A \cap\{x \in X \text { : there exists } f \in A \text { such that } f(x) \neq 0\} .
$$

If it exists, we will denote by $\partial A$ the Shilov boundary of $A$, that is, the minimal closed subset of $X$ with the property that each function in $A$ assumes its maximum on $\partial A$. On the other hand, it is said that $x_{0} \in X$ is a strong boundary point of $A$ if for each neighborhood $U$ of $x_{0}$, there is a function $f$ in $A$ such that $\left|f\left(x_{0}\right)\right|=\|f\|$ and $|f(x)|<\|f\|$ for all $x \in X-U$. We will denote by $\tau A$ the set of all strong boundary points of $A$.

We will denote by $\operatorname{Ch} A$ the Choquet boundary of $A$. Let us recall that each extreme point of the unit ball $V$ of the dual space of $A$ has the form $\mu e_{x}$, where $\mu$ is a complex number of modulus 1 and $e_{x}$ is the evaluation map at the point $x \in X$,

Received by the editors October 16, 1995.

1991 Mathematics Subject Classification. Primary 46E15; Secondary 46E25.

Research of the first author was supported in part by the Spanish Dirección General de Investigación Científica y Técnica (DGICYT, PS90-100).

Research of the second author was supported in part by Fundació Caixa Castelló, (A-39-MA). 
$e_{x}(f)=f(x)\left(f \in C_{0}(X)\right)$. The Choquet boundary for $A$ is defined as $\left\{x \in X: e_{x}\right.$ is a extreme point of $V\}$. Recall that although the Choquet boundary is usually defined in the case when $X$ is compact and $A$ separates points and contains the constants, both definitions agree in this case.

We say that a linear subspace $A$ of $C_{0}(X)$ is separating (resp. strongly separating) if given any pair of distinct points $x_{1}, x_{2}$ of $X$, then there exists $f \in A$ such that $f\left(x_{1}\right) \neq f\left(x_{2}\right)$ (resp. $\left.\left|f\left(x_{1}\right)\right| \neq\left|f\left(x_{2}\right)\right|\right)$. It is well-known that the Shilov boundary of a separating subalgebra of $C_{0}(X)$ always exists.

A separating (resp. strongly separating) linear subspace $A$ of $C_{0}(X)$ is said to be a separating (resp. strongly separating) function subspace if for all $x \in X$, there exists $f \in A$ such that $f(x) \neq 0$.

The source of this article is the classical Banach-Stone theorem. In its present form it states as follows: if there exists a linear isometry $T$ of $C_{0}(X)$ onto $C_{0}(Y)$, then there are a homeomorphism $h$ of $Y$ onto $X$ and a continuous map $a: Y \rightarrow \mathbb{K}$, $|a| \equiv 1$, such that $T$ can be written as a weighted composition map, that is,

$$
(T f)(y)=a(y) f(h(y)) \text { for all } y \in Y \text { and all } f \in C_{0}(X) .
$$

This well-known theorem has been generalized in several directions, for instance, by considering injective (not necessarily surjective) linear isometries. Perhaps the most important result of this type is due to Holsztyński [15]: if there exists a linear isometry $T$ of $C(X)$ into $C(Y)$, then we can find a closed subset $Y_{0}$ of $Y$ and a continuous map $h$ of $Y_{0}$ onto $X$ and a continuous map $a: Y_{0} \rightarrow \mathbb{K},|a| \equiv 1$, such that

$$
(T f)(y)=a(y) f(h(y)) \text { for all } y \in Y_{0} \text { and all } f \in C(X) .
$$

Some years before, Geba and Semadeni [14] had obtained an analogue of Holsztyński's theorem though for isotonic injective linear isometries. Also a number of applications of Holsztyński's theorem can be found in the literature. Recently, for instance, it has played a crucial role in the classification of isometric shift operators on $C(X)$ (see, e.g., [12] and [13]).

Generalizations of a similar type are provided by replacing $C_{0}(X)$ by its subspaces or subalgebras. Indeed, in 1948, Myers [20] proved that, if $\mathbb{K}=\mathbb{R}$, then a sufficient condition for $X$ and $Y$ to be homeomorphic is that a completely regular linear subspace of $C(X)$ and such a subspace of $C(Y)$ be isometrically isomorphic. Let us recall that a closed linear subspace $A$ of $C_{0}(X)$ is said to be completely regular if every $x \in X$ is a strong boundary point of $A$, i.e., $\tau A=X$.

In 1959, Nagasawa [21] (see also [11]) extended the Banach-Stone theorem for function algebras, that is, closed separating subalgebras with unit of $C(X)$-spaces. He proved that two function algebras are isomorphic as algebras if and only if they are isometric as Banach spaces.

In 1975, Novinger [22] went a step further and extended some of the above generalizations: if there exists a linear isometry $T$ from a linear subspace $A$ of $C(X)$ which is separating and contains the constants into $C_{0}(Y)$, then there are a continuous map $h$ of the Choquet boundary of $T(A), \operatorname{Ch} T(A)$, onto $\operatorname{Ch} A$ and a continuous map $a: \operatorname{Ch} T(A) \rightarrow \mathbb{K},|a| \equiv 1$, such that

$$
(T f)(y)=a(y) f(h(y)) \text { for all } y \in \operatorname{Ch} T(A) \text { and all } f \in A \text {. }
$$

Similar extensions of the Banach-Stone theorem have been given for subspaces of $C_{0}(X)$ equipped with different norms. Among these subspaces we point out 
the following: spaces of differentiable functions (see, e.g., [7]); spaces of absolutely continuous functions (see, e.g., [23]); spaces of Lipschitz functions (see, e.g., [10]).

If we weaken the geometric bond between $C_{0}(X)$ and $C_{0}(Y)$, the homeomorphism between $X$ and $Y$ may wither: Milutin [19] proved that if $X$ is any uncountable compact metric space (for instance, $X=[0,1] \cup\{2\}$ ), then $C(X)$ is linearly homeomorphic to $C([0,1])$. However, if the isometry is not weakened too much, good results can still be accomplished: Amir [1] and Cambern [6] proved that if $C_{0}(X)$ and $C_{0}(Y)$ are isomorphic under an isomorphim $T$ satisfying $\|T\| \cdot\left\|T^{-1}\right\|<2$, which is the best constant, then $X$ and $Y$ must also be homeomorphic. This theorem has been extended to cover various subspaces of $C_{0}(X)$-spaces, for instance, extremely regular subspaces (see, e.g., [5], [8], [9] or [16]) and function algebras (see, e.g., [17]).

The corresponding Banach-Stone theorem for $E$-valued continuous functions is not true even when the Banach space $E$ is the two dimensional space $\mathbb{R}^{2}$ and $X$, $Y$ are compact metric spaces (see [24]). Thus, the main concern in this line is to determine the geometric properties of $E$ which allow analogues of the Banach-Stone theorem. A systematic account of many of the generalizations in this and the above directions can be found in [4] or [18].

In this paper we deal with some of these generalizations. Indeed we focus on Holsztyński and Novinger's directions. Namely we study linear isometries of a strongly separating linear subspace $A$ of $C_{0}(X)$ into $C_{0}(Y)$ or onto such a subspace $B$ of $C_{0}(Y)$. We show that such isometries can be written as weighted composition maps on some subspaces of $Y$ ( $\sigma_{0} B$ for the onto case). Furthermore, under the onto assumption, we prove that $\sigma_{0} A$ and $\sigma_{0} B$ are homeomorphic. As straightforward consequences of this result we first show that the set of strong boundary points of $A$ and $B$ are homeomorphic. Also $\partial A$ and $\partial B$ are homeomorphic if $A$ and $B$ are assumed to be strongly separating function subspaces. We also provide an example which shows that this latter result may fail if the hypothesis " $A$ is strongly separating" is replaced by the weaker one " $A$ is separating".

Next we extend some results (op. cit.) by Amir and Arbel [3], Holsztyńsky [15], Myers [20] and Novinger [22]. We also apply our main results to study the isometries between separating function subalgebras of $C_{0}(X)$ and $C_{0}(Y)$ or, more generally, between semisimple commutative Banach algebras without unit and their Shilov boundaries.

Finally, we would like to remark that our techniques are not based on the usual concepts, such as extreme points of the unit ball of the dual of $C_{0}(X), T$-sets or $M$-ideals, used to prove the Banach-Stone theorem and their generalizations. We only use straightforward concepts instead.

\section{Some PREVIOUS LEMMAS}

In the sequel we will assume that every linear subspace $A$ of $C_{0}(X)$ has nonvoid Shilov boundary. Anyway, let us note that the Shilov boundary of a strongly separating linear subspace of $C_{0}(X)$ is nonvoid and coincides with the closure of its Choquet boundary ([2, Theorem 1$])$.

Lemma 2.1. Let $A$ be a linear subspace of $C_{0}(X)$. Then $\partial A=\sigma A$.

Proof. Let $x_{0} \in \partial A$. Given an open neighborhood $U$ of $x_{0}$, the closed set $X-U$ cannot be a boundary for $A$ since it does not contain $\partial A$. Consequently, there 
exists a function $f \in A$ which does not attain its maximum value on $X-U$, that is, $|f(x)|<\|f\|$ for all $x$ outside $U$.

Conversely, let $x_{0} \in \sigma A$. If $x_{0} \notin \partial A$, then there exists an open neighborhood $U$ of $x_{0}$ such that $\partial A \cap U=\emptyset$. Hence, there exists a function $f \in A$ not attaining its maximum value on $\partial A$, which contradicts the definition of boundary.

Lemma 2.2. Let $A$ be a linear subspace of $C_{0}(X)$. Let $T$ be a linear isometry from $A$ into $C_{0}(Y)$. Let $x \in X$ such that there exists $f \in A$ with $\|f\|=|f(x)|$. Let

$$
C_{x}:=\{f \in A: 1=\|f\|=|f(x)|\} .
$$

For any $f \in A$, let

$$
L(f):=\{y \in Y:\|T f\|=|(T f)(y)|\}
$$

and let $I_{x}:=\bigcap_{f \in C_{x}} L(f)$. Then $I_{x}$ is a nonempty subset of $Y$.

Proof. For any $f \in C_{x}$, we have

$$
I_{x} \subset M_{f}:=\{y \in Y:|(T f)(y)| \geq\|T f\| / 2\}
$$

and $M_{f}$ is compact because $T f \in C_{0}(Y)$. Hence, we only need to prove that if $f_{1}, \ldots, f_{n}$ belong to $C_{x}$, then $\bigcap_{i=1}^{n} L\left(f_{i}\right) \neq \emptyset$. We have that $1=\left\|f_{i}\right\|=\left|f_{i}(x)\right|$ for all $i=1, \ldots, n$. Let $f \in A$ be defined as

$$
\sum_{i=1}^{n}\left(\left|f_{i}(x)\right| / f_{i}(x)\right) f_{i} \text {. }
$$

Clearly $|f(x)|=n=\|f\|$. Since $T$ is an isometry, $\|T f\|=n$ and there is $y \in Y$ such that

$$
n=|(T f)(y)|=\sum_{i=1}^{n}\left|\left(\left|f_{i}(x)\right| / f_{i}(x)\right)\left(T f_{i}\right)(y)\right| .
$$

As $\left\|T f_{i}\right\| \leq 1$ for all $i=1, \ldots, n$, we deduce that $\left|\left(T f_{i}\right)(y)\right|=1$ for all $i=1, \ldots, n$, that is, $y \in \bigcap_{i=1}^{n} L\left(f_{i}\right)$.

Remark. Let $A$ be a linear subspace of $C_{0}(X)$ and let $x_{0} \in \partial A$. We then define the following subset of $Y$ :

$$
V_{x_{0}}:=\left\{y \in Y:|(T f)(y)|=\left|f\left(x_{0}\right)\right| \text { for all } f \in A\right\} .
$$

Lemma 2.3. Let $A$ be a linear subspace of $C_{0}(X)$ and let $x_{0} \in \sigma_{0} A$. Then $V_{x_{0}} \neq \emptyset$.

Proof. Let $f_{0} \in A$ such that $\left|f_{0}\left(x_{0}\right)\right|=1$. Given $\epsilon>0$, let

$$
U_{f_{0}, \epsilon}:=\left\{x \in X: 1-\epsilon<\left|f_{0}(x)\right|<1+\epsilon\right\} .
$$

Let $U$ be an open neighborhood of $x_{0}$. We will assume that $U \subseteq U_{f_{0}, \epsilon}$. Since $x_{0} \in \sigma_{0} A$ and $U$ is an open neighborhood of $x_{0}$, there exists a function $g_{0} \in A$ such that $\left\|g_{0}\right\|=1$ and $\left|g_{0}(x)\right|<1$ for all $x$ outside $U$. Since $(X \cup\{\infty\})-U$ is compact, we can consider

$$
s:=\sup _{x \in X-U}\left\{\left|g_{0}(x)\right|\right\}<1 .
$$

Then there exists $M>0$ such that $\left\|f_{0}\right\|+M s<1+\epsilon+M$. Take $x \in U$. Then

$$
\left|\left(f_{0}+M g_{0}\right)(x)\right| \leq 1+\epsilon+M \text {. }
$$

If $x \notin U$, then

$$
\left|\left(f_{0}+M g_{0}\right)(x)\right|<\left\|f_{0}\right\|+M s<1+\epsilon+M .
$$


As a consequence, $\left\|f_{0}+M g_{0}\right\|<1+\epsilon+M$. Hence, $\left\|T\left(f_{0}+M g_{0}\right)\right\|<1+\epsilon+M$. Furthermore, since

$$
\lim _{M \rightarrow+\infty} \frac{\left\|f_{0}+M g_{0}\right\|}{1+M}=1,
$$

we can choose $M$ in such a way that $\left|f_{0}+M g_{0}\right|$ attains its maximum value inside $U$. Otherwise,

$$
\lim _{M \rightarrow+\infty} \frac{\left\|f_{0}+M g_{0}\right\|}{1+M} \leq \lim _{M \rightarrow+\infty} \frac{\left\|f_{0}\right\|+M s}{1+M}=s<1,
$$

which is a contradiction. Thus, let $x_{1} \in U$ such that $\left\|f_{0}+M g_{0}\right\|=\left|\left(f_{0}+M g_{0}\right)\left(x_{1}\right)\right|$.

Let $x_{2} \in U$ such that $\left\|g_{0}\right\|=\left|g_{0}\left(x_{2}\right)\right|=1$. It is clear that we can choose $g_{0}$ such that $\left|\left(f_{0}+M g_{0}\right)\left(x_{2}\right)\right|=\left|f_{0}\left(x_{2}\right)\right|+M\left|g_{0}\left(x_{2}\right)\right|$. Thus,

$$
\left\|f_{0}+M g_{0}\right\| \geq\left|\left(f_{0}+M g_{0}\right)\left(x_{2}\right)\right| \geq M+1-\epsilon .
$$

Consequently,

$$
\left\|T f_{0}+M T g_{0}\right\| \geq M+1-\epsilon .
$$

From the definition of $I_{x_{1}}$ (see Lemma 2.2), we infer that

$$
\left\|T f_{0}+M T g_{0}\right\|=\left|\left(T f_{0}+M T g_{0}\right)\left(y_{1}\right)\right| \geq M+1-\epsilon
$$

for all $y_{1} \in I_{x_{1}}$. Since $\left|\left(T g_{0}\right)(y)\right| \leq 1$ for all $y \in Y$, we deduce that $\left|\left(T f_{0}\right)\left(y_{1}\right)\right| \geq 1-\epsilon$ for all $y_{1} \in I_{x_{1}}$.

Next we shall show that $\left|\left(T f_{0}\right)\left(y_{1}\right)\right| \leq 1+\epsilon$ for all $y_{1} \in I_{x_{1}}$. Let us define the function

$$
g_{1}:=\frac{f_{0}+M g_{0}}{\left\|f_{0}+M g_{0}\right\|} .
$$

Hence $\left|g_{1}\left(x_{1}\right)\right|=1=\left\|g_{1}\right\|$ and $\left|g_{1}\right|<1$ outside $U$. Since $(X \cup\{\infty\})-U$ is compact, we can consider

$$
r:=\sup _{x \in X-U}\left\{\left|g_{1}(x)\right|\right\}<1 .
$$

Arguing as above, we find a number $N \in \mathbb{K}$ such that $\left|f_{0}+N g_{1}\right|$ attains its maximum value inside $U$ and $\left\|f_{0}+N g_{1}\right\|<1+\epsilon+|N|$. Furthermore, we can choose $N$ in such a way that

$$
\left|\left(T f_{0}+N T g_{1}\right)\left(y_{1}\right)\right|=\left|\left(T f_{0}\right)\left(y_{1}\right)\right|+|N|\left|\left(T g_{1}\right)\left(y_{1}\right)\right| .
$$

As a consequence, since $\left|\left(T g_{1}\right)\left(y_{1}\right)\right|=1$, we have

$$
|N|+1+\epsilon \geq\left\|T f_{0}+N T g_{1}\right\| \geq\left|\left(T f_{0}+N T g_{1}\right)\left(y_{1}\right)\right|=\left|\left(T f_{0}\right)\left(y_{1}\right)\right|+|N|,
$$

that is, $\left|\left(T f_{0}\right)\left(y_{1}\right)\right| \leq 1+\epsilon$ for all $y_{1} \in I_{x_{1}}$. Gathering up the information we have obtained so far, it is clear that we can find a net $\left(x_{\alpha}\right)$ in $X$ converging to $x_{0}$ and a net $\left(y_{\alpha}\right)$ in $Y$ such that $y_{\alpha} \in I_{x_{\alpha}}$ for all $\alpha$ and such that there exists a subnet $\left(y_{\beta}\right)$ of $\left(y_{\alpha}\right)$ converging to some $y_{0} \in Y \cup\{\infty\}$ with $\left|\left(T f_{0}\right)\left(y_{0}\right)\right|=1$. This latter fact shows that $y_{0} \neq \infty$. Furthermore, it is apparent, from the above arguments, that, for any open neighborhood $V$ of $x_{0}$, there exist a term $x_{\beta_{0}}$ of the net $\left(x_{\beta}\right)$ and a function $g_{\beta_{0}} \in A$ such that $\left|g_{\beta_{0}}\left(x_{\beta_{0}}\right)\right|=1=\left\|g_{\beta_{0}}\right\|$ and $\left|g_{\beta_{0}}\right|<1$ outside $V$.

On the other hand, let $g \in A$ such that $g\left(x_{0}\right)=0$. We shall show that $(T g)\left(y_{0}\right)=$ 0 . Given $\epsilon>0$, let

$$
U_{g, \epsilon}:=\{x \in X:|g(x)|<\epsilon\} .
$$

Let $V$ be an open neighborhood of $x_{0}$ such that $V \subseteq U_{g, \epsilon}$. Hence, as mentioned above, there exist a term $x_{\beta_{0}}$ of the net $\left(x_{\beta}\right)$ and a function $g_{\beta_{0}} \in A$ such that $\left|g_{\beta_{0}}\left(x_{\beta_{0}}\right)\right|=1=\left\|g_{\beta_{0}}\right\|$ and $\left|g_{\beta_{0}}\right|<1$ outside $V$. Arguing as above, we find a 
number $P \in \mathbb{K}$ such that the function $\left|g+P g_{\beta_{0}}\right|$ attains its maximum value inside $V$ and $\left\|g+P g_{\beta_{0}}\right\|<\epsilon+|P|$. Furthermore, we can choose $P$ in such a way that

$$
\left|\left(T g+P T g_{\beta_{0}}\right)\left(y_{\beta_{0}}\right)\right|=\left|(T g)\left(y_{\beta_{0}}\right)\right|+|P|\left|\left(T g_{\beta_{0}}\right)\left(y_{\beta_{0}}\right)\right| \text {. }
$$

As a consequence, since $\left|\left(T g_{\beta_{0}}\right)\left(y_{\beta_{0}}\right)\right|=1$, we have

$$
|P|+\epsilon \geq \| T g+P T g_{\beta_{0}}|\geq|\left(T g+P T g_{\beta_{0}}\right)\left(y_{\beta_{0}}\right)|=|(T g)\left(y_{\beta_{0}}\right)|+| P \mid,
$$

that is, $\left|(T g)\left(y_{\beta_{0}}\right)\right| \leq \epsilon$. Therefore, since the net $\left(y_{\beta}\right)$ converges to $y_{0}$, we infer that $(T g)\left(y_{0}\right)=0$.

Let us now consider $l \in A$ such that $\left|l\left(x_{0}\right)\right|=1$. Let us define the function

$$
l^{\prime}:=\frac{f_{0}\left(x_{0}\right)}{l\left(x_{0}\right)} \cdot l
$$

and let $g \in A$ such that $l^{\prime}=f_{0}+g$. It is clear that $g\left(x_{0}\right)=0$. Consequently, by the above paragraph, $(T g)\left(y_{0}\right)=0$ and, since $l^{\prime}\left(x_{0}\right)=1$,

$$
\left|\left(T l^{\prime}\right)\left(y_{0}\right)\right|=\left|\left(T f_{0}\right)\left(y_{0}\right)+(T g)\left(y_{0}\right)\right|=1
$$

and, thus, $\left|(T l)\left(y_{0}\right)\right|=1$.

Finally, if $f \in A$, then we define $f^{\prime}=f /\left|f\left(x_{0}\right)\right|$. Hence $\left|f^{\prime}\left(x_{0}\right)\right|=1$. As a consequence, by the previous paragraph, we infer that $\left|\left(T f^{\prime}\right)\left(y_{0}\right)\right|=1$, i.e., $\left|f\left(x_{0}\right)\right|=$ $\left|(T f)\left(y_{0}\right)\right|$. The proof is complete.

Lemma 2.4. Let $A$ be a strongly separating linear subspace of $C_{0}(X)$ and let $T$ be a linear isometry from $A$ into $C_{0}(Y)$. If $x_{0}$ is a strong boundary point of $A$, then $V_{x_{0}}=I_{x_{0}}$

Proof. It suffices to check that $I_{x_{0}} \subseteq V_{x_{0}}$ since the other inclusion is apparent.

We will first show that, if $f \in A$ satisfies $f\left(x_{0}\right)=0$, then $(T f)(y)=0$ for all $y \in I_{x_{0}}$. Let us suppose that there exists $y_{0} \in I_{x_{0}}$ such that $(T f)\left(y_{0}\right) \neq 0$ and $f\left(x_{0}\right)=0$ for some $f \in A$. We will assume, without loss of generality, that $\|f\|=1$ and $(T f)\left(y_{0}\right)=\alpha>0$. Let

$$
U=\{x \in X:|f(x)| \geq \alpha / 2\} .
$$

Since $x_{0}$ is a strong boundary point of $A$ and $y_{0} \in I_{x_{0}}$, there exists $g \in A$ such that, multiplying by a constant if necessary, $\left|g\left(x_{0}\right)\right|=1=\|g\|,|g(x)|<1$ for all $x \in U$ and $(T g)\left(y_{0}\right)=1$. Since, from the definition of $C_{0}(X), U$ is a compact set, we can consider

$$
s:=\sup _{x \in U}\{|g(x)|\}<1 .
$$

Thus there is a real number $M>0$ such that $1+M s<\alpha+M$. We will distinguish two cases: If $x \in U$, then

$$
|(f+M g)(x)| \leq 1+M s
$$

If $x \notin U$, then

$$
|(f+M g)(x)| \leq \alpha / 2+M .
$$

That is, we have that $\|f+M g\|<\alpha+M$, but

$$
\alpha+M=(T f)\left(y_{0}\right)+(M T g)\left(y_{0}\right) \leq\|T(f+M g)\|,
$$

which is absurd since $T$ is an isometry.

Finally, let us suppose that there exists $y^{\prime} \in I_{x_{0}}$ such that $\left|(T f)\left(y^{\prime}\right)\right| \neq\left|f\left(x_{0}\right)\right|$ for some $f \in A$. Since $x_{0}$ is a strong boundary point of $A$, there will exist a function 
$k \in A$ such that $k\left(x_{0}\right)=1=\|k\|$. Hence it is straightforward to check that the function

$$
l(x):=f(x)-f\left(x_{0}\right) \cdot k(x)
$$

belongs to $A$ and, furthermore,

$$
l\left(x_{0}\right)=0
$$

and

$$
(T l)\left(y^{\prime}\right)=(T f)\left(y^{\prime}\right)-f\left(x_{0}\right) \cdot(T k)\left(y^{\prime}\right) \neq 0
$$

since $\left|(T k)\left(y^{\prime}\right)\right|=1$. This fact contradicts the paragraph above.

\section{INTO ISOMETRIES BETWEEN SUBSPACES}

Theorem 3.1. Let $T$ be a linear isometry of a strongly separating linear subspace $A$ of $C_{0}(X)$ into $C_{0}(Y)$. Then there are a subset $Y_{0}$ of $Y$, which is a boundary for $T(A)$, a continuous map $h$ from $Y_{0}$ onto $\sigma_{0} A$ and a continuous map a $: Y_{0} \rightarrow \mathbb{K}$, such that $|a(y)|=1$ for all $y \in Y_{0}$, and

$$
(T f)(y)=a(y) f(h(y)) \text { for all } y \in Y_{0} \text { and all } f \in A .
$$

Furthermore, if $\sigma_{0} A$ is compact, then $Y_{0}$ is closed.

Proof. Let $Y_{0}$ be the set $\bigcup_{x \in \sigma_{0} A} V_{x}$. It is clear, by Lemma 3.3, that $Y_{0}$ is nonvoid. In order to prove that $Y_{0}$ is a boundary for $T(A)$, let us suppose that there exists $f \in A$ such that

$$
|(T f)(y)|<\|T f\|=\|f\|
$$

for all $y \in Y_{0}$. Then we can find $x_{0} \in \sigma_{0} A$ such that

$$
\left|f\left(x_{0}\right)\right|=\|f\|=\|T f\| .
$$

Let $y_{0} \in V_{x_{0}}$. Then $y_{0} \in Y_{0}$ and

$$
\|f\|=\|T f\|=\left|f\left(x_{0}\right)\right|=\left|(T f)\left(y_{0}\right)\right|,
$$

which contradicts the above assumptions.

Next, we define the map $h$ of $Y_{0}$ onto $\sigma_{0} A$ as $h(y):=x$ if $y \in V_{x}$. Since $A$ is strongly separating, given $x, x^{\prime} \in \sigma_{0} A$ with $x \neq x^{\prime}$, it is easy to check that $V_{x} \cap V_{x^{\prime}}=\emptyset$. Thus the map $h$ is well-defined. Moreover, since $V_{x} \neq \emptyset$ for every $x \in \sigma_{0} A, h$ is onto.

In order to prove the continuity of $h$, suppose that $h\left(y_{0}\right)=x_{0}$ for some $y_{0} \in Y_{0}$. Let $f \in A$ such that $f\left(x_{0}\right)=1$. Hence, $\left|(T f)\left(y_{0}\right)\right|=1$. Let $\left(y_{\alpha}\right)$ be a net in $Y_{0}$ converging to $y_{0}$ and let $h\left(y_{\alpha}\right)=x_{\alpha}$ for all $\alpha$. Since $\left|(T f)\left(y_{0}\right)\right|=1$, we can assume, without loss of generality, that ||$(T f)\left(y_{\alpha}\right)|-1|<1 / 2$ for all $\alpha$. Then, from the definition of $V_{x_{\alpha}},\left|f\left(x_{\alpha}\right)\right|>1 / 2$ for all $\alpha$. Let $\left(x_{\beta}\right)$ be a subnet of $\left(x_{\alpha}\right)$ converging to $x_{1} \in X \cup\{\infty\}$. Consequently, $\left|f\left(x_{1}\right)\right| \geq 1 / 2$. Hence, $x_{1} \neq \infty$. If $x_{1} \neq x_{0}$, then we take $g \in A$ such that

$$
\left|g\left(x_{0}\right)\right|=1 \neq s=\left|g\left(x_{1}\right)\right| .
$$

Take a subnet $\left(y_{\gamma}\right)$ of $\left(y_{\beta}\right)$ such that

$$
||(T g)\left(y_{\gamma}\right)|-|(T g)\left(y_{0}\right)||<|1-s| / 2 .
$$

Hence

$$
|| g\left(h\left(y_{\gamma}\right)\right)|-1|<|1-s| / 2
$$

and $\left(\left|g\left(x_{\gamma}\right)\right|\right)$ does not converge to $\left|g\left(x_{1}\right)\right|$, which is a contradiction. Hence every subnet of $\left(x_{\alpha}\right)$ has a subnet that converges to $x_{0}$ and then we have that $\left(x_{\alpha}\right)$ converges to $x_{0}$. 
Now, let us define a map $a$ of $Y_{0}$ into $\mathbb{K}$ as follows: given $y \in Y_{0}$, let $f$ be any function in $A$ such that $f(h(y))=1$. Hence, we define $a(y):=(T f)(y)$ for all $y \in Y_{0}$. This is a well-defined map because if we take another function $g$ in $A$ such that $g(h(y))=1$, then $(f-g)(h(y))=0$ and by the definition of $h,(T f)(y)=(T g)(y)$.

On the other hand, it is clear that $|a(y)|=1$ for all $y \in Y_{0}$.

Next we prove both that $T$ can be written as a weighted composition map and, as a consequence, the continuity of $a$. We have already proved that if $f(h(y))=0$, then $(T f)(y)=0$ for all $y \in Y_{0}$ and all $f \in A$. If $f(h(y)) \neq 0$ for some $f \in A$ and some $y \in Y_{0}$, then let

$$
g=f-f(h(y)) k .
$$

$k$ being any function in $A$ such that $k(h(y))=1$. Clearly $g(h(y))=0$. Thus, $(T g)(y)=0$, that is,

$$
(T f)(y)=a(y) f(h(y)) .
$$

In order to prove the continuity of $a$, we will show that for each $y \in Y_{0}$, there exists an open neighborhood of $y$ where $a$ is continuous. Let us consider any $f \in A$ such that $f(h(y)) \neq 0$ and let

$$
W:=\left\{x \in \sigma_{0} A: f(x) \neq 0\right\} .
$$

It is clear that $h^{-1}(W)$ is an open neighborhood of $y$. Moreover the map $T f /(f \circ h)$ is continuous on $h^{-1}(W)$, and $a$ and $T f /(f \circ h)$ coincide on $h^{-1}(W)$.

Finally, assume that $\sigma_{0} A$ is compact and let $y_{0} \in Y$ such that there exists a net $\left(y_{\alpha}\right)$ in $Y_{0}$ converging to $y_{0}$. For all $\alpha, y_{\alpha}$ belongs to some $V_{x_{\alpha}}$ (see remark preceding Lemma 2.3) with $h\left(y_{\alpha}\right)=x_{\alpha} \in \sigma_{0} A$. Hence the net above has a subnet $\left(x_{\beta}\right)$ which converges to some $x_{0} \in \sigma_{0} A$. Since

$$
\left|f\left(x_{\beta}\right)\right|=\left|(T f)\left(y_{\beta}\right)\right|
$$

for all $\beta$ and all $f \in A$, we deduce, by the continuity of $f$ and $T f$, that

$$
\left|f\left(x_{0}\right)\right|=\left|(T f)\left(y_{0}\right)\right|
$$

for all $f \in A$, that is, $y_{0} \in V_{x_{0}} \subset Y_{0}$.

Remark. Theorem 3.1 generalizes Holsztyński's theorem by taking $X$ compact and $A=C(X)$.

Corollary 3.2. In the same conditions as in Theorem 3.1, $h$ sends $\operatorname{Ch} T(A)$ onto Ch $A$.

Proof. By [2, Theorem 1], we know that the Choquet boundary of $A$ is contained in $\partial A$. On the other hand, for every point $x$ of $\mathrm{Ch} A$, there exists $f \in A$ such that $f(x) \neq 0$, so $\mathrm{Ch} A \subset \sigma_{0} A$. Since $T^{-1}: T(A) \rightarrow A$ is a linear surjective isometry, we have that its adjoint $\left(T^{-1}\right)^{\prime}: A^{\prime} \rightarrow(T(A))^{\prime}$ sends the extreme points of the unit ball of $A^{\prime}$ onto such points of $(T(A))^{\prime}$. So, if $x \in \mathrm{Ch} A,\left(T^{-1}\right)^{\prime} e_{x}=\mu e_{y}$, where $\mu \in \mathbb{K},|\mu|=1$ and $y \in Y$. If $f \in A$,

$$
\begin{aligned}
|(T f)(y)| & =|\mu(T f)(y)| \\
& =\left|\mu e_{y}(T f)\right| \\
& =\left|\left(\left(T^{-1}\right)^{\prime} e_{x}\right)(T f)\right| \\
& =|f(x)| .
\end{aligned}
$$

We conclude that $y \in V_{x}$ and, consequently, that $\mathrm{Ch} A \subseteq h(\mathrm{Ch} T(A))$.

The other inclusion follows from the same arguments since $\left(T^{-1}\right)^{\prime}$ is bijective. 
Remark. It is evident, from Corollary 3.2, that Theorem 3.1 extends the main result (Theorem 1) of [22].

Theorem 3.3. Let $T$ be a linear isometry of a strongly separating linear subspace $A$ of $C_{0}(X)$ into $C_{0}(Y)$. Then $T f=E(a \cdot f \circ h)$, where $h$ is a continuous map defined from a subset $Y_{0}$ of $Y$ onto $\sigma_{0} A, a: Y_{0} \rightarrow \mathbb{K}$ is a continuous map such that $|a| \equiv 1$ and $E: Z \rightarrow C_{0}(Y)$ is a norm-preserving linear extension with $Z=$ $\{a \cdot f \circ h: f \in A\}$.

Proof. Let $a, h$, and $Y_{0}$ be as in Theorem 3.1 and let us define $E g:=T f$ being $g:=a \cdot f \circ h$ for some $f \in A$. Clearly this definition completes the proof.

\section{The onto CASE}

Theorem 4.1. Let $T$ be a linear isometry of a strongly separating linear subspace $A$ of $C_{0}(X)$ onto such a subspace $B$ of $C_{0}(Y)$. Then there exist a homeomorphism $h$ of $\sigma_{0} B$ onto $\sigma_{0} A$ and a continuous map $a: \sigma_{0} B \rightarrow \mathbb{K}$, such that $|a(y)|=1$ for all $y \in \sigma_{0} B$, and

$$
(T f)(y)=a(y) f(h(y)) \text { for all } y \in \sigma_{0} B \text { and all } f \in A .
$$

Proof. Let $h$ and $Y_{0}$ be as in Theorem 3.1. To prove the injectivity of $h$, we shall check that the sets $V_{x}$ are singletons. Suppose that $y_{0}, y_{1} \in V_{x}$ for some $x \in \sigma_{0} A$ and $y_{0} \neq y_{1}$. Consequently

$$
\left|(T f)\left(y_{0}\right)\right|=\left|(T f)\left(y_{1}\right)\right|=|f(x)|
$$

for all $f \in A$, which contradicts the strongly separating property of $B$.

We now show that $Y_{0}=\sigma_{0} B$. Let $y_{0} \in Y_{0}$. There exists $x_{0} \in \sigma_{0} A$ such that

$$
x_{0}=h\left(y_{0}\right) \text {. }
$$

Let $U$ be any open neighborhood of $y_{0}$. It falls out of the way we obtain $y_{0}$ in Lemma 2.3 that there exists a set $I_{x}$, for some $x \in X$, contained in $U$. This means, according to the definition of $I_{x}$ (see Lemma 2.1), that

$$
\bigcap_{f \in C_{x}} L(f) \subset U
$$

that is,

$$
\left(\bigcap_{f \in C_{x}} L(f)\right) \cap((X \cup\{\infty\}) \backslash U)=\emptyset .
$$

Thus we have an intersection of closed subsets whose intersection with the compact set $(X \cup\{\infty\}) \backslash U$ is empty. Hence, there exist finitely many functions $\left\{f_{1}, \ldots, f_{n}\right\} \subset$ $C_{x}$ such that

$$
\bigcap_{i=1}^{n} L\left(f_{i}\right) \subset U
$$

We can assume, with no loss of generality, that

$$
f_{i}(x)=1, \quad i=1, \ldots, n \text {. }
$$

Then the function $f:=\sum_{i=1}^{n} f_{i}$ satisfies $f(x)=n$. As a consequence,

$$
|(T f)(y)|<\|T f\|=n
$$

for all $y \notin U$, which implies that $y_{0} \in \sigma_{0} B$.

Conversely, to prove that $\sigma_{0} B \subset Y_{0}$, take $y_{0} \in \sigma_{0} B$. We now consider the inverse of $T$, which is an isometry of $B$ onto $A$. By Theorem 3.1, there exists a continuous 
map $k$ from a subset $X_{0}$ of $X$, defined as $\bigcup_{y \in \sigma_{0} B} V_{y}$, onto $\sigma_{0} B$. As above, we can prove that $X_{0} \subset \sigma_{0} A$. Let us consider $x_{0} \in X_{0}$ such that $k\left(x_{0}\right)=y_{0}$. It just remains to prove that $y_{0} \in V_{x_{0}}$. We know that, for all $g \in B$,

$$
\left|\left(T^{-1} g\right)\left(x_{0}\right)\right|=\left|g\left(y_{0}\right)\right| \text {, }
$$

that is, for all $f \in A$,

$$
\left|(T f)\left(y_{0}\right)\right|=\left|f\left(x_{0}\right)\right| .
$$

Consequently, $h\left(y_{0}\right)=x_{0}$. Hence, $h$ is a homeomorphism of $\sigma_{0} A$ onto $\sigma_{0} B$. Finally, by Theorem 3.1, $T$ is a weighted composition map.

Now the following corollary holds because of Theorem 4.1 and Corollary 3.2.

Corollary 4.2. In the same conditions as in Theorem 4.1, $h$ is a homeomorphism of $\mathrm{Ch} B$ onto $\mathrm{Ch} A$.

Remarks. 1. Let $T$ be the isometric embedding of $C_{0}(\mathbb{N})$ into $C(\mathbb{N} \cup\{\infty\})$. This example shows both that, in Theorem 3.1, $Y_{0}$ may not be closed and that, in Theorem 3.3, the Shilov boundaries of $A$ and $B$ are not homeomorphic in general. However, if $A$ and $B$ are assumed to be strongly separating function subspaces, then it is straightforward, from the definition of $\sigma_{0} A$ (resp. $\sigma_{0} B$ ) and by Lemma 2.1, that their Shilov boundaries are homeomorphic.

2. The following example shows that this latter assertion may fail if we replace the hypothesis " $A$ is strongly separating" by " $A$ is separating": Let us define the compact set

$$
X:=\left\{1-\frac{1}{n}: n \in \mathbb{N}\right\} \cup\left\{-1+\frac{1}{n}: n \in \mathbb{N}\right\} \cup\{-1,1\}
$$

and let $A^{\prime}$ be the set of all functions $f_{n} \in C(X), n=2,3,4, \ldots$, defined as follows: if $n$ is even,

$$
f_{n}(x):= \begin{cases}1 & \text { if } x=1-\frac{1}{n} \\ -1+\frac{1}{n} & \text { if } x=-1+\frac{1}{n} \\ 0 & \text { otherwise }\end{cases}
$$

If $n$ is odd,

$$
f_{n}(x):= \begin{cases}-1 & \text { if } x=-1+\frac{1}{n} \\ 1-\frac{1}{n} & \text { if } x=1-\frac{1}{n} \\ 0 & \text { otherwise. }\end{cases}
$$

Given $x \in X$ (resp. $x \in Y$ ), we will denote by $\chi_{x}$ the characteristic function of the singleton $\{x\}$. Let us define the function $f \in C(X)$ in the following way:

$$
f(x):=\left(\sum_{n=2}^{\infty} f_{n}(x)\right)+\chi_{1}-\chi_{-1}
$$

Let $A$ be the linear span of $A^{\prime}$ and $f$.

On the other hand, let us define the compact set

$$
Y:=\left\{\frac{1}{n}: n \in \mathbb{N}\right\} \cup\{0\}
$$

and let us define $g \in C(Y)$ as follows:

$$
g(x):=\left(\sum_{n=1}^{\infty} \chi_{\frac{1}{n}}\right)+\chi_{0} .
$$


Let $B$ be the linear span of the set $\left\{\chi_{\frac{1}{n}}: n \in \mathbb{N}\right\}$ and $g$. It is now a routine matter to verify that $A$ is a (not strongly) separating linear subspace of $C(X)$. Also, $A$ is linearly isometric to $B$. However,

$$
\partial A=\left\{1-\frac{1}{n}: n \text { even }\right\} \cup\left\{-1+\frac{1}{n}: n \text { odd }\right\} \cup\{-1,1\}
$$

is not homeomorphic to $\partial B=Y$.

3. The assertion of Theorem 4.1 cannot be strengthened to the effect " $X$ homeomorphic to $Y$ ". A counterexample is obtained by taking the isometry $T$ of $C_{0}(X)=C_{0}(0,1)$ into $C_{0}(Y)=C_{0}((0,1) \cup(1,2))$ defined to be $(T f)(x)=f(x)$ if $x \in(0,1)$, and $(T f)(x)=f(x-1) / 2$ if $x \in(1,2)$. Clearly $X$ is not homeomorphic to $Y$ because $Y$ is not connected.

Corollary 4.3. Let $T, X, Y, A$ and $B$ be as in Theorem 4.1. If, in addition, we assume that either $\tau A$ or $\tau B$ is a nonempty set, then $\tau A$ and $\tau B$ are homeomorphic.

Proof. Let us define the set

$$
Y_{00}=\bigcup_{x \in \tau A} V_{x} .
$$

By the definition of $h$ (see the proof of Theorem 3.1) and since it is injective (Theorem 4.1), we infer that $h\left(Y_{00}\right)=\tau A$. Hence, by virtue of Theorem 4.1, it suffices to check that $Y_{00}=\tau B$. Let $y_{0} \in Y_{00}$. There exists $x_{0} \in \tau A$ such that $x_{0}=h\left(y_{0}\right)$. Let $U$ be any open neighborhood of $y_{0}$. If $y \notin U$, then $y \in V_{x_{0}}$ since, by Lemma 2.4, $V_{x_{0}}=I_{x_{0}}$ whenever $x_{0}$ is a strong boundary point. Thus, by Lemma 2.2, there is $f_{y} \in A$ such that

$$
1=f_{y}\left(x_{0}\right)=\left\|f_{y}\right\|
$$

and

$$
\left|\left(T f_{y}\right)(y)\right|<1 .
$$

For each $y \in(Y \cup\{\infty\})-U$, we take an open neighborhood $U_{y}$ of $y$ such that $\left|\left(T f_{y}\right)\left(y^{\prime}\right)\right|<1$ for all $y^{\prime} \in U_{y}$. Since $(Y \cup\{\infty\})-U$ is compact, we can find $\left\{y_{1}, \ldots, y_{n}\right\} \subset(Y \cup\{\infty\})-U$ such that $(Y \cup\{\infty\})-U \subset \bigcup_{i=1}^{n} U_{y_{i}}$. Now, let us define the map

$$
g=\left(\sum_{i=1}^{n} f_{y_{i}}\right) / n
$$

It is clear that

$$
1=g\left(x_{0}\right)=\|g\|
$$

and

$$
1=\left|(T g)\left(y_{0}\right)\right|=\|T g\| .
$$

Moreover, $|(T g)(y)|<1$ for all $y$ outside $U$. Consequently, the elements of $Y_{00}$ are strong boundary points for $B$.

Conversely, let $y_{0} \in \tau B$. Arguing as in the preceding paragraph, we prove $X_{00} \subset \tau A$, where

$$
X_{00}:=\bigcup_{y \in \tau B} V_{y} .
$$

Thus, there exists $x_{0} \in \tau A$ such that $k\left(x_{0}\right)=y_{0}$, where $k$ is the inverse of $h$ (see the proof of Theorem 4.1). That is, $y_{0} \in V_{x_{0}} \subset Y_{00}$ and we are done. 
The following corollary shows that Myers' theorem ([20]) is valid also for noncompact spaces and complex valued functions. Moreover we can write the isometry as a weighted composition map.

Corollary 4.4. Let $T$ be a linear isometry of a completely regular linear subspace $A$ of $C_{0}(X)$ onto such a subspace $B$ of $C_{0}(Y)$. Then there exist a homeomorphism $h$ of $Y$ onto $X$ and a continuous map $a: Y \rightarrow \mathbb{K}$, such that $|a(y)|=1$ for all $y \in Y$, and

$$
(T f)(y)=a(y) f(h(y)) \text { for all } y \in Y \text { and all } f \in A .
$$

Proof. It is evident from the definition of completely regular linear subspace that $\sigma_{0} A=X$ and $\sigma_{0} B=Y$. The result follows now from Theorem 4.1.

Remark. It is clear that the linear isometry $T$ extends uniquely to an isometry from $C_{0}(X)$ onto $C_{0}(Y)$.

\section{Subspaces as quotient spaces}

Corollary 5.1. Let $T$ be a linear isometry of a strongly separating subspace $A$ of $C_{0}(X)$ into $C_{0}(Y)$. Then $\sigma_{0} A$ is homeomorphic to a quotient of a subspace $Y_{0}$ of $Y$.

Proof. With the same notation as in Theorem 3.1, let us define the following equivalence in $Y_{0}: y_{0} \sim y_{1}$ if $y_{0}, y_{1}$ belong to the same $V_{x}$, for some $x \in \sigma_{0} A$. If $\pi$ denotes the natural quotient map of $Y_{0}$ onto $\left(Y_{0} / \sim\right)$, then the map $h^{\sim}=h \circ \pi^{-1}$ is a continuous bijection of $\left(Y_{0} / \sim\right)$ onto $\sigma_{0} A$. To prove the continuity of $\left(h^{\sim}\right)^{-1}$, take a net $\left(x_{\alpha}\right)$ in $\sigma_{0} A$ converging to $x_{0} \in \sigma_{0} A$. For each $\alpha$, take $y_{\alpha} \in V_{x_{\alpha}}$. Clearly there exists a subnet $\left(y_{\beta}\right)$ of $\left(y_{\alpha}\right)$ converging to a point $y_{0} \in Y \cup\{\infty\}$. Take any $f \in A$. Then $\left(\left|f\left(x_{\beta}\right)\right|\right)$ converges to $\left|f\left(x_{0}\right)\right|$ and $\left|(T f)\left(y_{\beta}\right)\right|$ converges to $\left|(T f)\left(y_{0}\right)\right|$. As

$$
\left|(T f)\left(y_{\beta}\right)\right|=\left|f\left(x_{\beta}\right)\right|
$$

for all $\beta$, we have that

$$
\left|f\left(x_{0}\right)\right|=\left|(T f)\left(y_{0}\right)\right|
$$

for all $f \in A$, that is, $y_{0} \in V_{x_{0}}$. This implies that $\left(\left(h^{\sim}\right)^{-1}\left(x_{\beta}\right)\right)$ converges to $\left(h^{\sim}\right)^{-1}\left(x_{0}\right)$. In this way, every subnet of $\left(\left(h^{\sim}\right)^{-1}\left(x_{\alpha}\right)\right)$ has a subnet converging to $\left(h^{\sim}\right)^{-1}\left(x_{0}\right)$. So $\left(\left(h^{\sim}\right)^{-1}\left(x_{\alpha}\right)\right)$ converges to $\left(h^{\sim}\right)^{-1}\left(x_{0}\right)$. Then $\left(h^{\sim}\right)^{-1}$ is continuous.

Remark. Let us suppose that, in Corollary 5.1, $X$ is compact and $1 \in A$. We now consider the quotient space $Y / D$, where $D$ is a decomposition of $Y$ which consists of the subsets $V_{x}, x \in \sigma_{0} A$, and the singletons $\{y\}$ such that $y \in Y-Y_{0}$. Since now $a=T 1$ then $\|a\|=1$ and we can define an isometry $T^{\sim}$ of $A$ into $C_{0}(Y / D)$ by the requirement that $\left(T^{\sim} f\right)\left(y^{\sim}\right)=(\bar{a} T f)\left(\pi^{-1}\left(y^{\sim}\right)\right)$ for all $f \in A$ and all $y^{\sim} \in Y / D$, where $\bar{a}$ denotes the complex conjugate of the map $a$ and $\pi$ the natural quotient map of $Y$ onto $Y / D$. $T^{\sim}$ is well defined because, by Theorem 3.1, $\bar{a} T f$ is constant on each $V_{x}, x \in \sigma_{0} A$. As in Corollary 5.1, we prove that $\sigma_{0} A$ is homeomorphic to a subspace $(Y / D)_{0}$ of $Y / D$, defined like $Y_{0}$ in Theorem 3.1. Moreover, with the hypothesis of Corollary 5.1, there exists a norm-preserving linear extension $U$ from the subspace $\{A \circ h\}$ of $C\left(Y_{0}\right)$ into $C_{0}(Y)$ defined to be $U(g):=\bar{a}(T f)$, where $g:=f \circ h$ for some $f \in A$. 
All these remarks show that Corollary 5.1 extends a result by D. Amir and B. Arbel [3, Theorem 1], if we assume that $X$ is compact and $A=C(X)$. Furthermore, this assumption lets us claim that if $T(C(X))$ is a strongly separating linear subspace, then it is complemented in $C_{0}(Y)$. To prove this, we define a projection $\pi$ from $C_{0}(Y)$ onto $T(C(X))$ as follows: given $f \in C_{0}(Y)$, let $\pi(f)$ be

$$
T\left(\bar{a}\left(h^{-1}(-)\right)\right) \cdot f\left(h^{-1}(-)\right)
$$

where $h^{-1}$ is the inverse of $h$ defined as in Theorem 4.1. It is easy to check that $\pi \circ T=T$ and, consequently, $\pi^{2}=\pi$.

\section{IsOMetries BetWeEn SUbALGEBras}

Theorem 6.1. Let $T$ be a linear isometry of a separating function subalgebra $A$ of $C_{0}(X)$ onto such a subspace $B$ of $C_{0}(Y)$. Then

1.- $\partial A$ is homeomorphic to $\partial B$.

2.- There exists a continuous map $b: Y \rightarrow \mathbb{K}$ such that

$$
T(f g)(y)=b(y)(T f)(y)(T g)(y) \text { for all } y \in Y \text { and all } f, g \in A \text {. }
$$

Proof. 1.- It suffices to show that $A$ is strongly separating. Let $x_{1}, x_{2} \in X$ with $x_{1} \neq x_{2}$. There exists $f \in A$ such that $f\left(x_{1}\right)=z_{1}$ and $f\left(x_{2}\right)=z_{2}$ with $z_{1} \neq z_{2}$. If $\left|z_{1}\right|=\left|z_{2}\right|$, then we consider the function $g:=f+f^{2} \in A$. Hence, $g\left(x_{1}\right)=z_{1}\left(1+z_{1}\right)$ and $g\left(x_{2}\right)=z_{2}\left(1+z_{2}\right)$. With no loss of generality, we can assume that $\operatorname{Re} z_{1}$ and $\operatorname{Re} z_{2}$ are different. Otherwise we multiply the function $f$ by the complex number $i$ and, since $z_{1} \neq z_{2}$, we infer that $\operatorname{Re} i z_{1} \neq \operatorname{Re} i z_{2}$. Then

$$
\begin{aligned}
\left|g\left(x_{1}\right)\right|^{2} & =\left|z_{1}\right|^{2}\left|1+z_{1}\right|^{2} \\
& =\left|z_{1}\right|^{2}\left(1+z_{1}\right) \overline{\left(1+z_{1}\right)} \\
& =\left|z_{1}\right|^{2}\left(1+z_{1}+\overline{z_{1}}+\left|z_{1}\right|^{2}\right) \\
& =\left.\left|z_{1}\right|^{2}\left|1+2 \operatorname{Re} z_{1}+\right| z_{1}\right|^{2} \mid
\end{aligned}
$$

and

$$
\begin{aligned}
\left|g\left(x_{2}\right)\right|^{2} & =\left|z_{2}\right|^{2}\left|1+z_{2}\right|^{2} \\
& =\left|z_{2}\right|^{2}\left(1+z_{2}\right) \overline{\left(1+z_{2}\right)} \\
& =\left|z_{2}\right|^{2}\left(1+z_{2}+\overline{z_{2}}+\left|z_{2}\right|^{2}\right) \\
& =\left.\left|z_{2}\right|^{2}\left|1+2 \operatorname{Re} z_{2}+\right| z_{2}\right|^{2} \mid .
\end{aligned}
$$

Clearly, $\left|g\left(x_{1}\right)\right| \neq\left|g\left(x_{2}\right)\right|$. Summing up, $A$ is a strongly separating linear subspace of $C_{0}(X)$ and the result follows from Theorem 4.1.

2.- We know, by Theorem 4.1, that $a(y) T(f g)(y)=(T f)(y)(T g)(y)$ for all $f, g \in A$ and all $y \in \partial B$. Consequently, if $b$ denotes the complex conjugate of $a$,

$$
T(f g)(y)=b(y)(T f)(y)(T g)(y)
$$

for all $y \in \partial B$ and all $f, g \in A$.

Let $y_{0} \in Y-\partial B$ and let $f \in A$ such that $(T f)\left(y_{0}\right)=0$. We now show that $T(f g)\left(y_{0}\right)=0$ for all $g \in A$. It is clear that the maps 


$$
T(f g)(T k)(T k)
$$

and

$$
\left(T k^{2}\right)(T f)(T g)
$$

coincide on $\partial B$, for $k \in A$ such that $(T k)\left(y_{0}\right) \neq 0$. Thus they coincide on $Y$ and consequently $T(f g)\left(y_{0}\right)=0$.

To extend $b$ from $\partial B$ to $Y$, take, for each $y \in Y-\partial B, f, g$ in $A$ such that $(T f)(y) \neq 0$ and $(T g)(y) \neq 0$. Then we define

$$
b(y)=T(f g)(y) /(T f)(y)(T g)(y) .
$$

This extension of the map $b$ to the whole $Y$ is well defined because if we consider $k, l \in A$ with $(T k)(y) \neq 0$ and $(T l)(y) \neq 0$, then

$$
T(f g)(T k)(T l)
$$

and

$$
(T f)(T g) T(k l)
$$

coincide on $\partial B$ and, as a consequence, on $Y$. The continuity of $b$ follows from the continuity of $T(f g), T f$ and $T g$ in an open neighborhood of each $y$.

Theorem 6.2. Let $A, B$ be semisimple commutative Banach algebras (not necessarily with unit), such that $\|f\|^{2}=\left\|f^{2}\right\|$ for all $f \in A$ (resp. $f \in B$ ). If $T$ is a linear isometry of $A$ into (resp. onto) $B$, then there exists a continuous map (resp. a homeomorphism) $h$ of a subset $Y_{0}$ of the maximal ideal space $Y$ of $B$ (resp. the Shilov boundary $\partial B$ ) onto the Shilov boundary, $\partial A$, of $A$ and a continuous map $a: Y_{0} \rightarrow \mathbb{K}$ (resp. $\left.a: \partial B \rightarrow \mathbb{K}\right)$ with $|a(y)|=1$ for all $y \in Y_{0}$ (resp. $y \in \partial B$ ) and

$$
(T f)(y)=a(y) f(h(y)) \text { for all } y \in Y_{0}(\text { resp. } \partial B) \text { and all } f \in A .
$$

Proof. Since $\|f\|^{2}=\left\|f^{2}\right\|$ for all $f \in A$ (for all $f \in B$ respectively), the Gelfand transform is an isometry of $A$ (resp. $B$ ) into $C_{0}(X)$ (resp. $C_{0}(Y)$ ), where $X$ (resp. $Y$ ) is the maximal ideal space of $A$ (resp. $B$ ). We can, therefore, regard $A$ and $B$ as separating function subalgebras of $C_{0}(X)$ and $C_{0}(Y)$ respectively, and the result follows from Theorem 3.1 and Theorem 4.1.

Corollary 6.3. (Nagasawa) Two semisimple commutative Banach algebras with unit $A$ and $B$ such that $\|f\|^{2}=\left\|f^{2}\right\|$ for all $f \in A$ (resp. $f \in B$ ) are isometric as Banach spaces if and only if they are isomorphic as algebras.

Proof. Let us first regard $A$ and $B$ as in the proof of Theorem 6.2 and let $T$ be a linear isometry of $A$ onto $B$. It is clear, since both subalgebras have unit 1, that, in this context, the continuous function $a$ which appears in Theorem 6.1 is $T 1$. That is, $a \in B$. Furthermore, since $T$ is onto, there exists $f \in A$ such that $T f=1$. Finally, from Theorem 6.1 , we infer both that $b=a^{-1} \in B$ and that $b \cdot T$ is the desired algebra isomorphism.

The converse is clear.

Remark. As a consequence of Theorem 6.2 we deduce, for example, that the Shilov boundary of $H^{\infty}$, i.e. the space of bounded analytic functions on the open unit disk, is homeomorphic to the Shilov boundary of the algebra $H_{0}^{\infty}$ consisting of all $f \in H^{\infty}$ that vanish at the origin. This falls immediately out of the linear isometry 
$f \hookrightarrow z \cdot f$ of $H^{\infty}$ onto $H_{0}^{\infty}$. This example also shows that $H^{\infty}$ and $H_{0}^{\infty}$, despite being isometric as Banach spaces, are not algebraically isomorphic since $H_{0}^{\infty}$ does not have a unit. Consequently we cannot strengthen Corollary 6.3 to the effect " $A$ and $B$ are algebraically isomorphic", that is, Nagasawa's result for uniform algebras with unit (see [21]).

The authors wish to thank the referee for making several suggestions which improved this paper.

\section{REFERENCES}

[1] D. Amir, On isomorphisms of continuous function spaces, Israel J. Math. 3 (1966), 205-210. MR 34:516

[2] J. Araujo and J. J. Font, On Šilov boundaries for subspaces of continuous functions. To appear in Topology Appl.

[3] D. Amir and B. Arbel, On injections and surjections of continuous function spaces, Israel J. Math. 15 (1973), 301-310. MR 53:1239

[4] E. Behrends, M-structure and the Banach-Stone theorem, Lecture Notes in Mathematics 736, Berlin-Heidelberg-New York, Springer (1979). MR 81b:46002

[5] Y. Benyamini, Small into isomorphisms between spaces of continuous functions, Proc. A.M.S. 83 (1981), 479-485. MR 82j:46033

[6] M. Cambern, On isomorphisms with small bound, Proc. A.M.S. 18 (1967), 1062-1066. MR 36:669

[7] M. Cambern and V.D. Pathak, Isometries of spaces of differentiable functions, Math. Japonica 26 (1981), 253-260. MR 82h:46043

[8] B. Cengiz, A generalization of the Banach-Stone theorem, Proc. A.M.S. 40 (1973), 426-430. MR 47:9258

[9] H. B. Cohen, A bound-two isomorphism between $C(X)$ Banach spaces, Proc. A.M.S. 50 (1975), 215-217. MR 52:1279

[10] K. DeLeeuw, Banach spaces of Lipschitz functions, Studia Math. 21 (1961), 55-66. MR 25:4341

[11] K. DeLeeuw, W. Rudin and J. Wermer, The isometries of some functions spaces, Proc. A.M.S. 11 (1960), 694-698. MR 22:12380

[12] F. O. Farid and K. Varadarajan, Isometric shift operators on $C(X)$, Can. J. Math. 46 (1994), 532-542. MR 95d:47034

[13] A. Gutek, D. Hart, J. Jamison and M. Rajagopalan, Shift operators on Banach spaces, J. Funct. Anal. 101 (1991), 97-119. MR 92g:47046

[14] K. Geba and Z. Semadeni, Spaces of continuous functions (V), Studia Math. 19 (1960), 303-320. MR 22:8313

[15] H. Holsztyński, Continuous mappings induced by isometries of spaces of continuous functions, Studia Math. 26 (1966), 133-136. MR 33:1711

[16] K. Jarosz, Into isomorphisms of spaces of continuous functions, Proc. Amer. Math. Soc. 90 (1984), 373-377. MR 85k:46024

[17] K. Jarosz, Perturbations of Banach algebras, Lecture Notes in Math. 1120, Springer-Verlag, (1985). MR 86k:46074

[18] K. Jarosz and V. D. Pathak, Isometries and small bound isomorphisms of function spaces, Lecture Notes in Pure and Appl. Math. 136, Dekker (1992), 241-271. MR 93b:47061

[19] A.A. Miljutin, Isomorphisms of spaces of continuous functions on compacta of the power of the continuum (Russian), Teor. Funkcii Funkcional Anal. i Prilozhen. 2 (1966), 150-156. MR 34:6513

[20] S. B. Myers, Banach spaces of continuous functions, Ann. of Math. 49 (1948), 132-140. MR 9:291c

[21] M. Nagasawa, Isomorphisms between commutative Banach algebras with application to rings of analytic functions, Kodai Math. Sem. Rep. 11 (1959), 182-188. MR 22:12379

[22] W. P. Novinger, Linear isometries of subspaces of continuous functions, Studia Math. 53 (1975), 273-276. MR 54:5818 
[23] V.D. Pathak, Linear isometries of absolutely continuous functions, Can. J. Math. 34 (1982), 298-306. MR 83f:46023

[24] K. Sundaresan, Spaces of continuous functions into a Banach space, Studia Math. 48 (1973), 15-22. MR 48:9377

Departamento de Matemáticas, Estadística y Computación, Universidad de Cantabria, Facultad de Ciencias, Avda. de los Castros, s. N., E-39071 Santander, Spain

E-mail address: araujoj@ccaix3.unican.es

Departamento de Matemáticas, Universitat Jaume I, Campus Penyeta Roja, E-12071 Castellón, Spain

E-mail address: font@mat.uji.es 